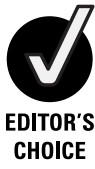

Additional materials are published online only. To view these files please visit the journal online (http://dx.doi.org/ 10.1136/thoraxjnl-2012201747)

${ }^{1}$ Respiratory Unit, Great Ormond Street Hospital for Children NHS Foundation Trust, London, UK ${ }^{2}$ Portex Respiratory Unit, UCL Institute of Child Health, London,

${ }^{3}$ Department of Paediatric Respiratory Medicine, Imperial College \& Royal Brompton \& Harefield Hospital NHS

Foundation Trust, London, UK ${ }^{4}$ Department of Paediatric Respiratory Medicine, Barts \& The London Children's Hospital, London, UK

${ }^{5}$ Queen Mary's Hospital for Children, Epsom \& St Helier University Hospitals NHS Trust Surrey, UK

${ }^{6}$ Neonatal Unit, Homerton University Hospital NHS

Foundation Trust, Barts \& The London School of Medicine and Dentistry, Queen Mary University of London, London,

${ }^{7}$ Department of Paediatric Respiratory Medicine, Kings College Hospital, London, UK ${ }^{8}$ Department of Child Health, Lewisham Healthcare NHS Trust, London, UK

${ }^{9}$ Centre for Paediatric Epidemiology \& Biostatistics, UCL Institute of Child Health, London, UK

\section{Correspondence to}

Dr Ah-Fong Hoo, Respiratory

Unit, Great Ormond Street Hospital for Children NHS

Foundation Trust, London WC1N 3JH, UK:

a.hoo@ucl.ac.uk

Received 10 February 2012 Accepted 25 May 2012

Published Online First 29 June 2012

\title{
Lung function is abnormal in 3-month-old infants with cystic fibrosis diagnosed by newborn screening
}

\author{
Ah-Fong Hoo, ${ }^{1}$ Lena P Thia, ${ }^{2}$ The Thanh Diem Nguyen, ${ }^{2}$ Andrew Bush, ${ }^{3}$ Jane Chudleigh, \\ Sooky Lum, ${ }^{2}$ Deeba Ahmed, ${ }^{2}$ lan Balfour-Lynn, ${ }^{3}$ Siobhan B Carr, ${ }^{4}$ \\ Richard J Chavasse, ${ }^{5}$ Kate L Costeloe, ${ }^{6}$ John Price, ${ }^{7}$ Anu Shankar, ${ }^{8}$ Colin Wallis, \\ Hilary A Wyatt, ${ }^{7}$ Angela Wade, ${ }^{9}$ Janet Stocks, ${ }^{2}$ on behalf of the London Cystic \\ Fibrosis Collaboration (LCFC)
}

\begin{abstract}
Background Long-term benefits of newborn screening (NBS) for cystic fibrosis (CF) have been established with respect to nutritional status, but effects on pulmonary health remain unclear.
\end{abstract}

Hypothesis With early diagnosis and commencement of standardised treatment, lung function at $\sim 3$ months of age is normal in NBS infants with CF.

Methods Lung clearance index (LCI) and functional residual capacity (FRC) using multiple breath washout (MBW), plethysmographic (pleth) FRC and forced expirations from raised lung volumes were measured in 71 infants with CF (participants in the London CF Collaboration) and 54 contemporaneous healthy controls age $\sim 3$ months.

Results Compared with controls, and after adjustment for body size and age, $\mathrm{LCl}, \mathrm{FRC}_{\mathrm{MBW}}$ and $\mathrm{FRC}_{\text {pleth }}$ were significantly higher in infants with CF (mean difference (95\% Cl): 0.5 (0.1 to 0.9), $p=0.02 ; 0.4$ (0.1 to 0.7 ) $\mathrm{p}=0.02$ and 0.9 (0.4 to 1.3), $\mathrm{p}<0.001$, z-scores, respectively), while forced expiratory volume $\left(\mathrm{FEV}_{0.5}\right)$ and flows $\left(\mathrm{FEF}_{25-75}\right)$ were significantly lower $(-0.9$ $(-1.3$ to -0.6$), p<0.001$ and $-0.7(-1.1$ to -0.2$)$, $p=0.004$, z-scores, respectively). $21 \%(15 / 70)$ of infants with CF had an elevated LCI (>1.96 z-scores) and $25 \%$ (17/68) an abnormally low $\mathrm{FEV}_{0.5}$ (below -1.96 z-scores). While only eight infants with CF had abnormalities of $\mathrm{LCl}$ and $\mathrm{FEV}_{0.5}$, using both techniques identified abnormalities in 35\% (24/68). Hyperinflation $\left(\right.$ FRC $_{\text {pleth }}>1.96$ z-scores) was identified in $18 \%(10 / 56)$ of infants with CF and was significantly correlated with diminished $\mathrm{FEF}_{25-75}(r=-0.43, p<0.001)$ but not with $\mathrm{LCl}$ or $\mathrm{FEV}_{0.5}$

Conclusion Despite early diagnosis of CF by NBS and protocol-driven treatment in specialist centres, abnormal lung function, with increased ventilation inhomogeneity and hyperinflation and diminished airway function, is evident in many infants with CF diagnosed through NBS by 3 months of age.

\section{INTRODUCTION}

The prognosis of cystic fibrosis (CF) has improved dramatically over the years due to implementation of aggressive treatment to optimise nutrition and pulmonary health following diagnosis and increasing global uptake of newborn screening (NBS) for CF.

\section{Key messages}

What is the key question?

- Is lung function normal at 3 months of age in infants with cystic fibrosis (CF) diagnosed via newborn screening?

What is the bottom line?

- Despite early diagnosis of CF by newborn screening and protocol-driven treatment in specialist centres, abnormal lung function is evident in many screened infants with CF by 3 months of age.

\section{Why read on?}

- This study, the largest of its kind and the only one with contemporaneous healthy controls, describes early lung development in newborn screened infants with CF.

Despite convincing evidence of the long-term benefits of NBS for CF with respect to improved nutritional status, ${ }^{12}$ the extent to which pulmonary outcomes have improved remains controversial Although some studies have failed to demonstrate any benefits with screening, ${ }^{3}$ others have reported significantly less pulmonary disease on chest radiography ${ }^{4}$ and stable lung function (LF) with less marked decline over time in the screened group. ${ }^{5-8}$ The Australian Respiratory Early Surveillance Team for Cystic Fibrosis (AREST-CF) group initially reported normal values of forced expired volume in $0.5 \mathrm{~s}\left(\mathrm{FEV}_{0.5}\right)$ in NBS infants with $\mathrm{CF}$ in the first 6 months of life, ${ }^{9}$ whereas a more recent report using slightly different methodology found significant reductions in forced expired flows and volumes during the same period. ${ }^{10}$

Even in the absence of any clinical respiratory symptoms, infants with CF diagnosed clinically have impaired LF shortly after diagnosis ${ }^{11} 12$ and this impairment persists into school age despite treatment in specialist CF centres. ${ }^{13-15}$ We have previously shown that the lung clearance index (LCI) measured by multiple breath washout (MBW) is a more sensitive measure of early lung disease in preschool and young school-age children with CF than spirometry, ${ }^{16-18}$ and that 
measurements during the preschool years are highly predictive of LF at 6-10 years of age. ${ }^{13}$ During infancy, complementary information is provided if the forced expiratory manoeuvres ${ }^{19}$ and $\mathrm{MBW}$ are undertaken. ${ }^{20}$ Nevertheless, the extent to which infant LF tests should be used to help guide CF management during infancy remains controversial. ${ }^{21}$

Since universal CF NBS was implemented in the UK in October 2007, the median age of CF diagnosis has fallen to $\sim 1$ month. ${ }^{22}$ However, there have yet to be any large randomised controlled trials of treatment in this age group. For these to be initiated, it is vital to have a greater understanding of the natural history of lung disease in such infants. This study aimed to determine baseline LF within the first 3 months of age in NBS infants with CF and compare findings to prospectively recruited healthy infants of similar age. We hypothesised that, with early diagnosis and commencement of standardised specialist treatment, there would be no difference in LF by 3 months of age in NBS infants with CF compared with healthy babies.

\section{METHODS}

As part of a longitudinal collaborative research program of infants with CF diagnosed by NBS (see online supplement), this study received ethical approval (\#09/HO71/314) from the North Thames Multi-Centre Research Ethics Committee (REC) and the local RECs of the participating specialist centres.

NBS infants with CF born between January 2009 and July 2011 were eligible for recruitment to the London CF Collaboration (LCFC). Healthy full-term infants of similar age were recruited prospectively from a London community (for details, including eligibility criteria, see online supplement, section 2). Lung function tests (LFTs) at 3 months were completed.

\section{Study protocol}

\section{Clinical data}

With parental consent, participating centres prospectively completed case record forms (CRFs) at diagnosis and each subsequent clinic visit, documenting mode and date of diagnosis, presentation, genotype, history of respiratory symptoms and/or infection, microbiology, treatment, somatic growth and additional investigations undertaken for clinical purposes. These forms enabled auditing and tracking of participating CF centres' adherence to a standardised study treatment protocol (online supplement, section 6) in accordance to the UK CF Trust guidelines. $^{23}$

Following diagnosis, all infants were commenced on multivitamins, pancreatic supplements if appropriate, and prophylactic flucloxacillin according to the standardised study treatment protocol (online supplement, section 6). Cough swabs were taken every 2-3 months at clinics and whenever symptomatic, using a standardised protocol for collection, storage and analysis of samples. ${ }^{24}$

\section{Lung function testing and anthropometry}

All infants were tested at Great Ormond Street Hospital/UCL Institute of Child Health at $\sim 3$ months postnatal age, when clinically stable and at least 3 weeks after any respiratory tract illness. Infants were weighed and examined, oxygen saturation $\left(\mathrm{SpO}_{2}\right)$ levels were measured (Masimo Radical-7 pulse oximeter, Irvine, California, USA) and vital signs assessed prior to administering chloral hydrate orally $(60-100 \mathrm{mg} / \mathrm{kg}$ ). Weight and crown-heel length were expressed as z-scores to adjust for age and sex. ${ }^{25}$ LFTs were performed during epochs of quiet sleep with the child lying supine. Heart rate and $\mathrm{SpO}_{2}$ were moni- tored continuously throughout testing. Infant urine or maternal saliva samples were collected for cotinine assay to validate parental reported smoking habits (online supplement, section 5, table E4). Parents provided informed written consent and were present throughout measurements.

LCI, a measure of ventilation inhomogeneity, and functional residual capacity ( $\mathrm{FRC}_{\mathrm{MBW}}$ ) were measured with $\mathrm{MBW}$, using a respiratory mass spectrometer and customised software. ${ }^{20}$ The Jaeger BabyBody device (V.4.65; Care Fusion, San Diego, California, USA) was used to measure plethysmographic FRC $\left(\mathrm{FRC}_{\mathrm{pleth}}\right){ }^{26} 27$ total respiratory compliance $\left(\mathrm{C}_{\mathrm{rs}}\right)$ and resistance $\left(\mathrm{R}_{\mathrm{rs}}\right){ }^{28}$ forced expiratory volume $\left(\mathrm{FEV}_{0.5}\right)$, forced vital capacity (FVC) and forced expiratory flows $\left(\mathrm{FEF}_{75}, \mathrm{FEF}_{25-75}\right)$ from an inflation pressure of $30 \mathrm{cmH}_{2} \mathrm{O}$ using the raised volume technique. $^{19}$ Measurements were always performed in that order. Details of data collection and analysis as described previously $^{20} 27-30$ are summarised in the online supplement. Results were expressed as z-scores to adjust for body size, sex and age if appropriate using reference equations derived from up to 140 healthy white infants studied in our department over the past decade using identical equipment and protocols ${ }^{19} \quad 3132$ (online supplement, section 3). Abnormal lung function was defined as results outside the $95 \%$ limits of normal (ie, below 1.96 z-scores for $\mathrm{FEV}_{0.5}$ or $>1.96$ z-scores for LCI $(<2.5$ th or $>97.5$ th centile respectively).

\section{Statistical analysis and power of study}

Comparisons of group differences were performed using student $t$ tests for continuous variables (age, body size and LFT z-scores) or $\chi^{2}$ analyses for categorical variables (sex, ethnicity, maternal smoking, parental occupation and asthma) (PASW Statistics V.18). Within the CF group, the relationship between LFT outcomes was quantified using Pearson correlations. Multiple linear regression analyses were used to quantify impact of maternal smoking (data not shown) and the extent to which potential clinical determinants (CF genotype, respiratory symptoms, cough swab culture and antibiotic use) are associated with LFT outcomes within the CF group after adjustment for sex and current body size. Model estimates and differences between groups are presented with $95 \%$ CIs. Taking into account two primary outcomes (LCI and $\mathrm{FEV}_{0.5}$ ) a sample size of 70 infants with CF and 50 controls (equivalent to 58 in each group) will allow detection of differences equivalent to 0.58 or 0.66 z-scores at the $5 \%$ significance level with $80 \%$ or $90 \%$ power, respectively. ${ }^{33}$ Statistical significance was taken as $\mathrm{p}<0.05$.

\section{RESULTS}

During the study period, 110 infants screened positive for CF, of whom successful LFT measurements were obtained in 79 (81\% of those eligible). Inspection of the prospective CRFs, and regular discussion with consultants, suggested that the standardised treatment protocol had been adhered to in all infants at the time of testing. Details are summarised in figure 1, including success rates for each LFT. For clarity, the results presented here are limited to those without meconium ileus $(n=71)$, although including such infants did not affect the results (data not shown). Of the 274 families with potentially eligible healthy infants whom we contacted, 39 (14\%) were ineligible. Of the remaining 235, 54 (23\% of those eligible) attended for LFTs (figure 1, details in figure $\mathrm{E} 1$, online supplement).

Median (IOR) age at diagnosis for the CF infants was 3.6 (3.1-4.4) weeks; the majority were either homozygous $(59 \%)$ or heterozygous (32\%) $\Delta \mathrm{F} 508$ and $8(11 \%)$ were pancreatic 

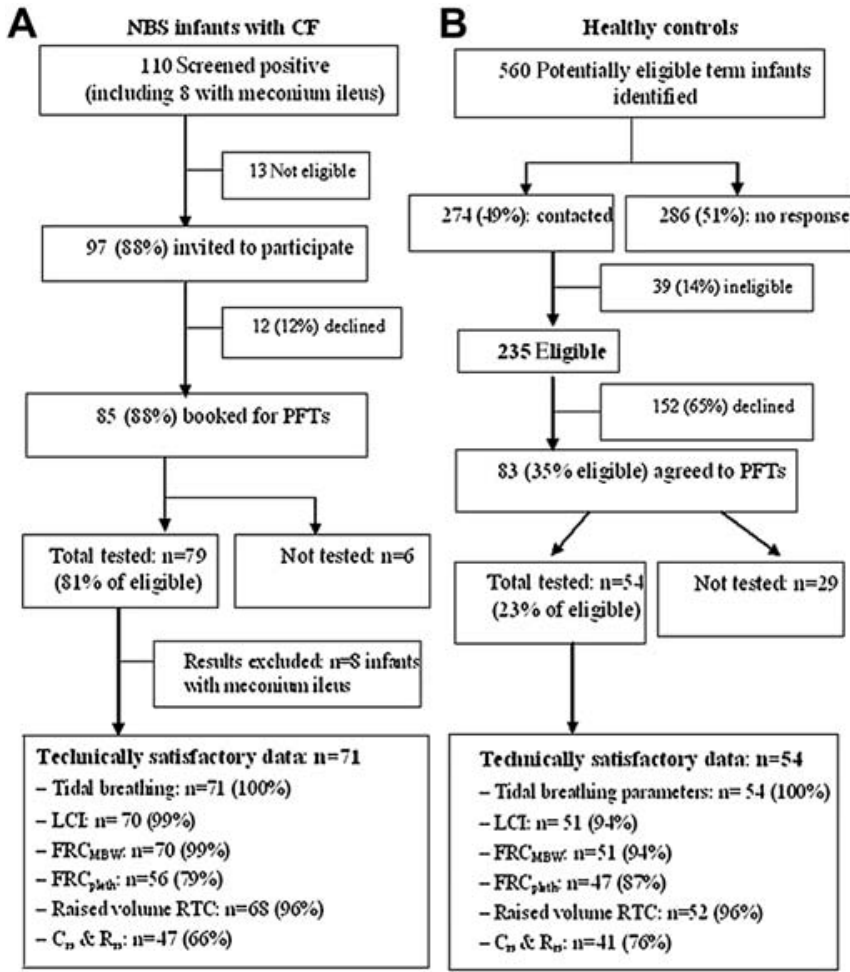

Figure 1 Flow diagram showing success rates in relation to recruitment and achievement of technically acceptable infant lung function outcomes in NBS infants with CF (A) and healthy controls (B). $\mathrm{CF}$, cystic fibrosis; $\mathrm{C}_{\mathrm{rs}}$, total respiratory compliance; $\mathrm{FRC}$, functional residual capacity; LCl, lung clearance index; MBW, multiple breath washout; NBS, newborn screening; PFT, pulmonary function test; pleth, plethysmographic; $\mathrm{R}_{\mathrm{rs}}$, total respiratory resistance; $\mathrm{RTC}$, Rapid Thoracoabdominal Compression.

sufficient. Sixty five (92\%) NBS infants with CF had had no respiratory or only mild coryzal symptoms prior to LFTs (figure 2). The remaining 6 (8\%) had had significant cough and/ or wheeze, 16 (23\%) had had at least one positive cough swab by $\sim 3$ months of age (two with Pseudomonas aeruginosa; 10 with Staphylococcus aureus and four with Haemophilus influenzae). These children were prescribed antibiotics according to a standardised treatment protocol (online supplement, section 6) in addition to the prophylactic flucloxacillin prescribed for all infants on diagnosis. No infant had positive cough swab culture for Stenotrophomonos maltophilia; Burkholderia cepacia complex or Aspergillus fumigatus. Additional antibiotics were also prescribed for 36 infants with negative cough swabs, 32 (89\%) of whom had had prior cough and/or wheeze. Thus in total, 52 (73\%) NBS infants with CF received additional antibiotics prior to their first LFTs at $\sim 3$ months (figure 2 ), but all were asymptomatic at the time of testing. Of the 46 infants with CF who had cough swabs taken within 10 days of LFTs, only three yielded positive growth (two for $S$ aureus and one for $H$ influenzae).

Table 1 summarises background characteristics of study participants. With the exception of a slightly lower, but statistically significant, gestational age, both groups were very similar.

Infant details at the time of LFTs are shown in table 2. NBS infants with CF were on average 1 week younger than healthy controls, with a significantly greater proportion having birth weights $<10$ th percentile. After taking age and sex into account, infants with CF were significantly lighter and shorter and had a lower body mass index than the controls. The change in

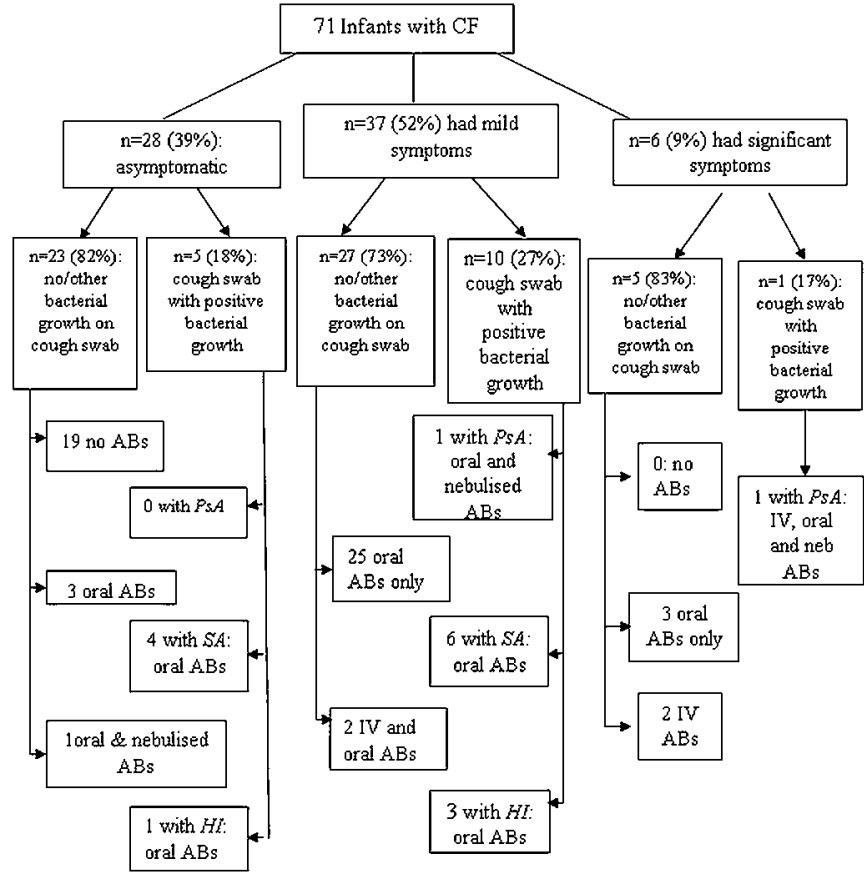

Figure 2 Clinical symptoms and additional antibiotic treatment* of newborn screened infants with cystic fibrosis (CF) prior to lung function tests. Although mothers of infants with CF occasionally reported mild symptoms (slight cough or mild snuffles) in the weeks prior to LFTs, on the day of the test all infants had clear chests on auscultation with no sign of blocked nostrils or cough. Reports of significant symptoms included previous wheeze, crackles, tachypnoea with or without cough and cold. *Antibiotic prescribed in addition to prophylactic flucloxacillin due to respiratory symptoms and/or positive growth on cough swab. See online supplement for standardised treatment protocol. $A B$, antibiotic; HI, Haemophilus influenzae; IV, intravenous; PsA, Pseudomonas aeruginosa; SA, Staphylococcus aureus.

weight z-score between birth and LFT was significantly lower in infants with CF than in controls (table 2).

\section{Lung function results}

Technically satisfactory measurements of LCI and FRC $_{M B W}$ were obtained in 121 infants (97\% of those in whom they were attempted), forced expired volumes and flows in 120 (96\%), plethysmographic FRC in $103(82 \%), \mathrm{C}_{\mathrm{rs}}$ and $\mathrm{R}_{\mathrm{rs}}$ in $88(70 \%)$ (figure 1, table 3 ). Failures were largely due to infants waking prior to completion of the test protocol or technically unacceptable data. With the exception of tidal volume, which was slightly higher (0.4 z-scores) in those with CF, there were no significant differences for any of the tidal breathing outcomes or passive respiratory mechanics $\left(\mathrm{C}_{\mathrm{rs}}\right.$ and $\left.\mathrm{R}_{\mathrm{rs}}\right)$. LCI was significantly higher in those with CF, whether expressed as absolute values (online supplement, table E2) or as z-scores (table 3, figure 3). Similarly after adjustment for age, length and sex, FRC $_{M B W}$ was significantly higher $(0.38 \mathrm{z}$-score) in infants with CF. Additional evidence of hyperinflation and gas trapping was seen in those with CF, with $\mathrm{FRC}_{\text {pleth }}$ and $\triangle \mathrm{FRC}$ (ie, difference between $\mathrm{FRC}_{\text {pleth }}$ and $\mathrm{FRC}_{\mathrm{MBW}} \mathrm{z}$-scores) being significantly elevated (table 3 , figure 3). After correction for body size, forced expired flows and volumes were significantly decreased in infants with CF compared with healthy controls, by between 0.5 and 0.9 z-scores, indicating the presence of airways obstruction (table 3 , figure 3 ). The extent to which interpretation of these results would have differed had they simply been 
Table 1 Comparison of background characteristics in infants with cystic fibrosis* and healthy controls

\begin{tabular}{|c|c|c|c|c|}
\hline & Infants with $\mathrm{CF}^{*}(\mathrm{n}=71)$ & Healthy controls $(n=54)$ & $\Delta(95 \% \mathrm{Cl}), \mathrm{CF}-$ controls & p Value \\
\hline Boy, n (\%) & $33(46)$ & $27(50)$ & $-4 \%(-21 \%$ to $13 \%)$ & 0.696 \\
\hline Gestational age, weeks & $39.3(1.5)$ & $40.2(1.2)$ & $-0.9(-1.4$ to -0.5$)$ & $<0.001$ \\
\hline Birth weight, z-score $†$ & $-0.34(0.96)$ & $0.01(0.84)$ & $-0.35(-0.67$ to -0.03$)$ & 0.035 \\
\hline White mother, n (\%) & $62(87)$ & $47(87)$ & $0 \%(-12 \%$ to $13 \%)$ & 0.962 \\
\hline Maternal smoking during pregnancy, $\mathrm{n}(\%)$ & $8(11)$ & $4(7)$ & $4 \%(-7 \%$ to $14 \%)$ & 0.456 \\
\hline Current maternal smoking, n (\%) & $9(13)$ & $5(9)$ & $4 \%(-8 \%$ to $15 \%)$ & 0.540 \\
\hline Maternal asthma, $\mathrm{n}(\%)$ & $13(18)$ & $10(19)$ & $0 \%(-14 \%$ to $13 \%)$ & 0.976 \\
\hline
\end{tabular}

Data shown as $\mathrm{n}(\%)$ for categorical variables and mean (SD) for continuous variables.

* Limited to those without meconium ileus.

†Calculated using UK WHO algorithms. ${ }^{25}$

$\S \mathrm{n}=69 \mathrm{CF}$ and $\mathrm{n}=53$ control infants.

$\mathrm{CF}$, cystic fibrosis; $\Delta$, difference between groups.

expressed as absolute values or weight-corrected ratios is presented in table E2 of the online supplement.

\section{Additional determinants of lung function}

On multivariable analyses, after adjustment for CF, other potential determinants (sex, gestational age, birth weight z-score, pre- or postnatal maternal smoking and maternal asthma) were not significantly associated with any LF z-scores. Among infants with CF, with the exception of a significantly lower $\mathrm{FEV}_{0.5}$ (mean $(95 \% \mathrm{CI}$ ): -0.70 ( -1.29 to -0.10$)$ z-scores) in those who had received any additional antibiotics for symptoms or positive cough swab, there was no significant association between LF outcomes and the infants' genotype, clinical status or treatment prior to LFTs at $\sim 3$ months of age (see table E3, OLS).

\section{Relationship between different lung function outcomes in infants with CF}

The relationship between selected LF outcomes in infants with $\mathrm{CF}$ is shown in figure E2 (online supplement). There was no significant relationship between the two primary outcomes $\mathrm{FEV}_{0.5}$ and LCI $(r=-0.10, \mathrm{p}=0.432)$. Twenty-one percent of infants had an LCI above $1.96 \mathrm{z}$-scores, whereas $25 \%$ had an $\mathrm{FEV}_{0.5}$ below -1.96 z-scores, while only $12 \%(8 / 68)$ had abnormalities detected by both these tests (figure E2a); if based on either test, $35 \%(24 / 68)$ would be identified with abnormal results. $\mathrm{FEF}_{25-75}$ and $\mathrm{FEV}_{0.5}(\mathrm{r}=0.73, \mathrm{p}<0.001)$ detected a similar proportion of infants outside the normal range $(24 \%$ and $25 \%$, respectively, figure $\mathrm{E} 2 \mathrm{~b}$ ), whereas $\mathrm{FEF}_{75}$ was less discriminative (only detecting abnormalities in 15\% of infants; data not shown). FRC pleth $\mathrm{Z}$-score and $\triangle \mathrm{FRC}$ were highly correlated $(\mathrm{r}=0.66, \mathrm{p}<0.001$, figure $\mathrm{E} 2 \mathrm{~d})$, both detecting a similar proportion of infants with abnormally elevated results $(18 \%$ and $20 \%$, respectively). Forty-four percent (31/71) of NBS infants with CF had at least one abnormal result if based on $\mathrm{LCI}, \mathrm{FEV}_{0.5}$ or FRC $_{\text {pleth. }}$.

\section{DISCUSSION}

In this, the largest study of its kind and the only one with contemporaneous healthy controls, we have shown that, by 3 months of age, many NBS infants with CF have reduced forced expired flows and volumes, abnormal gas mixing and hyperinflation, despite early diagnosis and protocol-driven management, which included prophylactic oral flucloxacillin from time of diagnosis.

\section{Strengths and weaknesses}

The major strengths of this study include the fact that all lung function measurements were performed to international standards in a single centre by a highly experienced team, thereby minimising methodological or analytical bias. Accurate identification of the extent to which abnormalities in LF were present in individual infants was facilitated by expressing results as $z$-scores. These were derived from equipment-specific reference equations based on a large group of healthy infants studied in our department with the same equipment and methods over the past decade. ${ }^{31} 32$ The ability to recruit a large group of contemporaneous healthy controls specifically for this study further strengthened the confidence with which we could detect changes due to CF lung disease after adjusting for other relevant determinants such as body size, age and sex. ${ }^{34} 35$

Table 2 Infant details at time of lung function test

\begin{tabular}{|c|c|c|c|c|}
\hline & Infants with CF (n=71) & Healthy controls $(n=54)$ & $\Delta(95 \%$ Cl) CF - controls & p Value \\
\hline Age at test*, weeks & $11.4(2.3)$ & $12.2(2.0)$ & $-0.82(-1.59$ to -0.06$)$ & 0.043 \\
\hline Weight, kg & $5.32(0.84)$ & $6.05(0.78)$ & $-0.73(-1.02$ to -0.45$)$ & $<0.001$ \\
\hline Weight, z-score $†$ & $-0.75(1.08)$ & $0.06(1.93)$ & $-0.81(-1.17$ to -0.45$)$ & $<0.001$ \\
\hline Length, $\mathrm{cm}$ & $59.2(2.7)$ & $61.6(2.3)$ & $-2.4(-3.3$ to -1.7$)$ & $<0.001$ \\
\hline Length, z-score $†$ & $-0.14(1.04)$ & $0.72(0.86)$ & $-0.86(-1.19$ to -0.52$)$ & $<0.001$ \\
\hline Body mass index & $15.1(1.6)$ & $15.9(1.4)$ & $-0.8(-1.3$ to -0.3$)$ & 0.005 \\
\hline Body mass index, z-score $\dagger$ & $-0.94(1.08)$ & $-0.47(0.98)$ & $-0.47(-0.84$ to -0.10$)$ & 0.013 \\
\hline
\end{tabular}

Unless stated otherwise, data presented as mean (SD).

*Postnatal age at test corrected for gestational age.

†Calculated using UK WHO algorithms. ${ }^{25}$

$\mathrm{CF}$, cystic fibrosis; $\Delta$, mean difference between groups. 
Table 3 Comparison of lung function in 71 infants with cystic fibrosis and 54 healthy controls

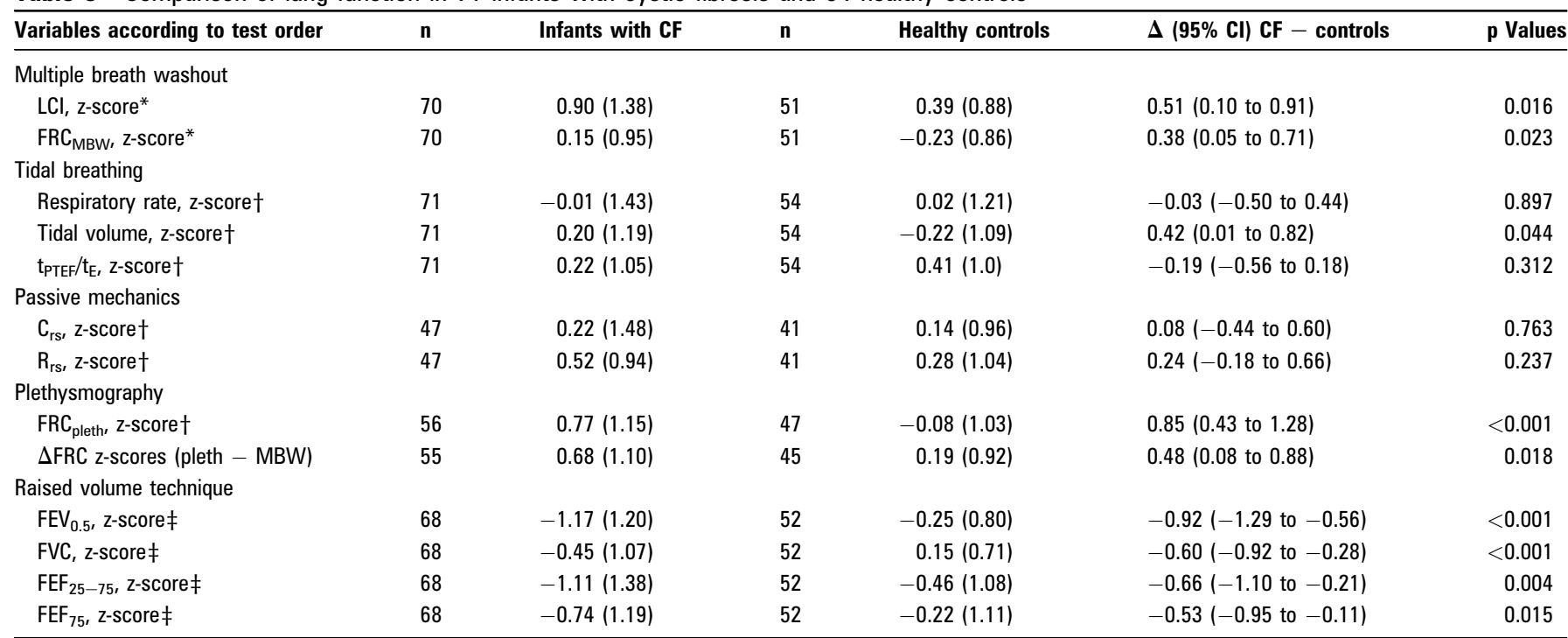

For comparison with previous publications, results expressed as absolute values, or in relation to body weight, are presented in table E2, online supplement.

Data shown as mean (SD).

*According to Lum et al. ${ }^{31}$

†According to Nguyen et al. ${ }^{32}$

$\ddagger$ According to Lum et al. ${ }^{34}$

$\mathrm{CF}$, cystic fibrosis; $\mathrm{Cl}$, confidence interval of the difference; $\Delta$, mean difference between groups.

$\mathrm{CF}$, cystic fibrosis; $\mathrm{C}_{\mathrm{rs}}$, total respiratory compliance; $\mathrm{FEF}_{75}$, forced expiratory flow at $75 \%$ of vital capacity; $\mathrm{FEF}_{25-75}$, forced expiratory flow between $25 \%$ and $75 \%$ of vital capacity; $\mathrm{FEV}_{0.5}$ forced expiratory volume in $0.5 \mathrm{~s}$; FRC, functional residual capacity; FVC, forced vital capacity; LCl, lung clearance index; MBW, multiple breath washout; pleth, plethysmographic; $\mathrm{R}_{\mathrm{rs}}$, total respiratory resistance; $\mathrm{TPTEF} / \mathrm{tE}$, ratio of the time to reach peak tidal expiratory flow in relation to expiratory time.

Inevitably, it was necessary to approach a large number of families of healthy infants to recruit appropriate numbers for comparison, with only $25 \%$ of those eligible and whom we could contact actually attending for the tests. This could potentially introduce some bias, especially as due to data protection issues, it was not possible to document background data from those not responding or agreeing to participate. However, it was reassuring to find no significant differences with respect to potential determinants of infant LF such as ethnicity, maternal history of asthma, socioeconomic status (based on parental occupation) or exposure to tobacco smoke between the controls and infants with CF, suggesting that the group were representative of the local population. Furthermore, when expressed as z-scores, both anthropometry and LF were very similar in this current healthy control group as in those previously recruited in London by our team. ${ }^{15} 20$ Travel expenses were reimbursed, but no incentives were provided to encourage attendance for either group, thereby removing a potential source of bias. Infants with CF were, on average, born 1 week earlier than the controls, a pattern that we have noted in previous studies. ${ }^{11}$ Any potential group differences due to developmental changes were avoided by studying all infants within a narrow age range; mean difference at time of test being less than a week.

Of the 110 infants screening positive for CF over the 2.5-year recruitment period, $12 \%$ were excluded due to coexistent morbidity or psychosocial reasons, with tests not possible in a further five infants before 4 months of age due to recent exacerbations and deferment of appointments. If anything, the results from this study therefore underestimate the degree of morbidity by 3 months of age. Nevertheless, with only $13 \%$ of parents declining participation (including one family that withdrew after initial consent), we were able to test 79/110 $(72 \%)$ of the entire cohort and $81 \%$ of those eligible.

Since lung function was assessed in the first few months of life, we excluded results from those presenting with meconium ileus to preclude any adverse effects related to surgery, although subsequent sub-analysis indicated that the results were not affected by including such children. All infants were tested at least 3 weeks after a respiratory illness when free of symptoms, in an attempt to minimise impact of acute exacerbations. This did mean that we had to exclude several healthy controls in whom it was impossible to rearrange appointments within the designated age range for this study of 'early LFT' (figure E1, online supplement). While it could be argued that the observed differences in LF between CF and controls may simply represent post-respiratory tract infection changes in those with CF, this is unlikely given the lack of association between LF and respiratory symptoms in those with CF, except for a significant reduction in z-FEV $\mathrm{F}_{0.5}$ when infants had received additional antibiotics (table E3, online supplement).

To optimise recruitment to this observational study and to ensure initial LFTs could be undertaken within the first months of life, routine bronchoscopy and bronchoalveolar lavage were not included within this study protocol but, together with chest high-resolution CT scans, will be included when these children are reassessed at 1 year of age.

A further strength of this study was the wide range of tests applied, minimising the chance that early changes in LF would be missed. As in a previous study of infants, ${ }^{20}$ but in contrast to findings in older children, ${ }^{13} 161736$ we found that assessments of lung disease based on forced expiratory manoeuvres and ventilation inhomogeneity were necessary to identify early lung disease during the first year of life, with additional useful information regarding hyperinflation being obtained from plethysmographic lung volumes. The slightly lower absolute mean LCI observed in infants with CF in this study (table E2, online supplement) than in our previous study ${ }^{20}$ probably reflects the fact that these infants were diagnosed by NBS rather than clinically, and were therefore assessed earlier in the disease process. Had assessments been limited to a single technique, 

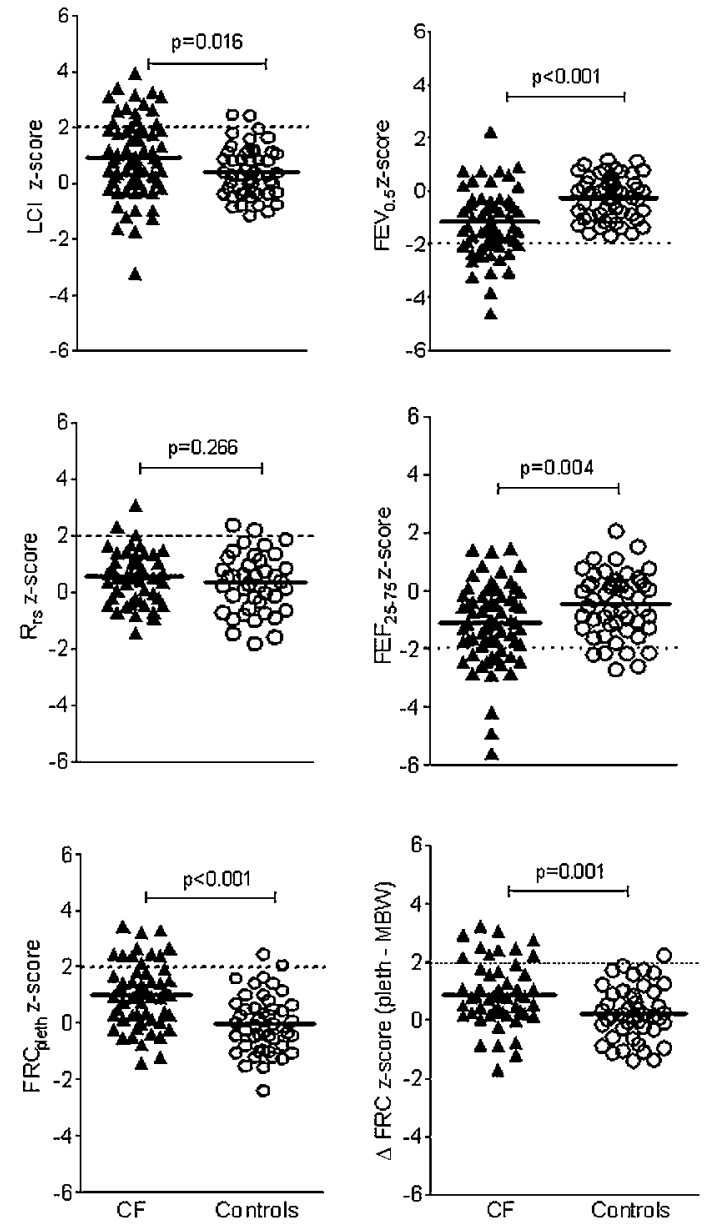

Figure 3 Comparison of lung function outcomes between infants with cystic fibrosis and healthy controls. As expected, approximately $95 \%$ of healthy controls had values within \pm 1.96 z-scores, with none having an abnormal $\mathrm{FEV}_{0.5} \mathrm{z}$-score, 2/51 (4\%) having an elevated LCl z-score, and 2/47 (4\%) an elevated $\mathrm{FRC}_{\text {pleth }} \mathrm{z}$-score. $\mathrm{FEF}_{25-75}$, forced expiratory flow between $25 \%$ and $75 \%$ of vital capacity; $\mathrm{FEV}_{0.5}$, forced expiratory volume in $0.5 \mathrm{~s} ; \mathrm{FRC}_{\text {pleth, }}$ plethysmographic functional residual capacity; $\mathrm{LCl}$, lung clearance index; MBW, multiple breath washout; $R_{r s}$, total respiratory resistance.

abnormalities would only have been detected in $\sim 25 \%$ of infants with CF, this proportion rising to $35 \%$ when using either of the two primary outcomes ( $\mathrm{LCI}$ and $\mathrm{FEV}_{0.5}$ ). The relatively poor correlation between these various outcomes suggests that they are reflecting different aspects of underlying pathophysiology. Despite their relative simplicity, assessments of tidal breathing variables and passive mechanics were far less discriminatory than the other techniques and could be usefully omitted when investigating infants with $\mathrm{CF}^{37}$ While $\mathrm{C}_{\mathrm{rs}}$ results were lower and $\mathrm{R}_{\mathrm{rs}}$ higher in those with $\mathrm{CF}$ when expressed as absolute values (table E2, online supplement), this was largely related to body size at the time of testing; $\mathrm{C}_{\mathrm{rs}}$ increasing and $\mathrm{R}_{\mathrm{rs}}$ decreasing with somatic growth. ${ }^{32} 38$ In contrast to all other outcome measures investigated, after adjusting for length and/or age, no significant differences were observed with respect to either $\mathrm{C}_{\mathrm{rs}}$ or $\mathrm{R}_{\mathrm{rs}}$ (table 3 ).

\section{Clinical status}

Despite early diagnosis and commencement of pancreatic enzyme replacement therapy, vitamin supplement and prophylactic antibiotics, infants with CF experienced significantly slower growth during the first few months and were significantly lighter and shorter than their healthy peers by the time of the 3-month LFTs. Furthermore, by this age, $61 \%$ of the screened infants had had some respiratory symptoms (52\% mild, 9\% severe), $23 \%$ a positive cough swab and $73 \%$ had received antibiotics in addition to their routine prophylactic medication. Pulmonary involvement is known to be present early, with some infants with CF having evidence of inflammation in the bronchoalveolar lavage fluid as early as 4 weeks of age. ${ }^{39-41}$ With the exception of a significantly lower $\mathrm{FEV}_{0.5}$ in those who had received additional antibiotics for symptoms or positive cough swab, there was no significant association between LF outcomes and the infant's genotype, clinical status, growth trajectory or treatment prior to the LFTs at 3 months of age. Of note, many infants who had been treated aggressively for respiratory exacerbations in the first few months had entirely normal LF by 3 months, whereas others with no prior symptoms or cause for concern had evidence of early lung disease.

\section{Comparison with the literature}

The commonest characteristics of LF abnormalities described in CF lung disease during the first years of life have been airway obstruction detected using the raised volume technique, ${ }^{10-121520}$ hyperinflation indicated by elevated resting lung volumes, ${ }^{42}$ increased ventilation inhomogeneity ${ }^{20}$ and, in infants and slightly older children, gas trapping, ${ }^{17} 184344$ all of which were observed in this study by 3 months of age in infants diagnosed by NBS.

In a US multicentre evaluation of LF in infants with CF aged 4-24 months (21\% diagnosed by screening), ${ }^{42}$ elevated lung volumes and diminished forced expiratory flows, but no reduction in $\mathrm{FEV}_{0.5}$, were reported when compared with historical controls. The variability in skill mix and experience of the laboratories participating in that study, together with the lack of contemporaneous healthy controls and different age range studied, may have contributed to differences in findings compared with the current results. ${ }^{35}$

The Australian AREST-CF study recently published LF results from infants with CF diagnosed by NBS. ${ }^{9}{ }^{10}$ When using a lung inflation pressure of $20 \mathrm{cmH}_{2} \mathrm{O}$ during forced expiratory manoeuvres, LF was reported to be normal during the first 6 months of life, but thereafter declined at a rapid rate. ${ }^{9}$ However, the number of infants studied during the first months of life was limited. By contrast, a more recent publication from this group, ${ }^{9} 10$ reporting measurements from 28 NBS infants with CF within the first 6 months of life when using an inflation pressure of $30 \mathrm{cmH}_{2} \mathrm{O}$ as recommended by the American Thoracic Society/European Respiratory Society guidelines ${ }^{19}$ showed diminished LF at the time of the first test, with continued deterioration over the next 2 years of life when the results were compared with published reference equations. ${ }^{45}$

\section{Clinical implications}

The results from this study indicate that despite early diagnosis and rapid implementation of therapy, including prophylactic antibiotics, a substantial number of NBS infants with CF have abnormalities of LF within the first 3 months of life. The apparent wellness of the cohort should not lead to complacency, and prompt and aggressive treatment of any abnormal symptoms or signs is surely vital. Follow-up of this cohort will be essential to ascertain the extent to which early changes in LF persist throughout the first year of life; if there is catch-up growth with conventional treatment, then novel, molecular- 
based therapies ${ }^{46} 47$ may be safely deferred; if not, there will be a compelling case for initiating treatment early in these infants, using physiological endpoints to detect benefit.

\section{CONCLUSIONS}

Despite early diagnosis of CF by NBS and protocol-driven treatment in specialist centres, abnormal LF, with increased ventilation inhomogeneity and hyperinflation and diminished airway function, is evident in many infants with CF diagnosed through NBS by 3 months of age. CF clinicians should not be lulled into thinking that babies with CF identified by NBS have good pulmonary health in the first few months of life.

Acknowledgements We thank the infants and parents who participated in this study and contributions by members of the London CF Collaboration (including Janet Stocks, Andy Bush, John Price, Colin Wallis, Ranjan Suri, Paul Aurora, lan BalfourLynn, Siobhan Carr, Caroline Pao, Hilary Wyatt, Gary Ruiz, Richard Chavesse, Anu Shanker, Wanda Kozlowska, Ah-Fong Hoo, Sooky Lum, The Thanh Diem Nguyen, Lena Thia, Jane Chudleigh, and Ammani Prasad). We also thank Per Gustafsson for advice and assistance regarding the multiple breath inert gas washout technique.

Contributors Conception and design of the study: JS, AB. Supervision of the study: JS. Research governance issues including ethics committee approval: JS, JC. Setting up of recruitment process: AFH, JC. Technical training, supervision and audit of data collection/analyses: AFH. Recruitment of infants with CF: AB, IBL, SBC, HAW, JP, RJC, AS. Recruitment of healthy control infants: KLC, AFH, DA, LPT, TDN, JC. Data collection/analysis and interpretation of results: JS, AFH, LPT, TDN, JC, SL. Statistical analyses: AFH, JS, AW. Drafting the manuscript: AFH, JS, LPT, IBL, AB, CW. Approval for intellectual content: all authors.

Funding This study is supported by grants from the Cystic Fibrosis Trust, UK; Special Trustees: Great Ormond Street Hospital for Children, London, UK; Smiths Medical Ltd, UK; Comprehensive Local Research Network, UK. It was also supported by the NIHR Respiratory Disease Biomedical Research Unit at the Royal Brompton and Harefield NHS Foundation Trust and Imperial College London.

Competing interests None.

Patient consent Obtained.

Ethics approval Ethics approval was granted by the North Thames Multi-centre Research Ethics Committee (REC) (\#09/H071/314) and local REC of the participating specialist centres.

Provenance and peer review Not commissioned; externally peer reviewed.

\section{REFERENCES}

1. Farrell PM, Lai HJ, Li Z, et al. Evidence on improved outcomes with early diagnosis of cystic fibrosis through neonatal screening: enough is enough! J Pediatr 2005;147 S30-6.

2. Koscik RL, Farrell PM, Kosorok MR, et al. Cognitive function of children with cystic fibrosis: deleterious effect of early malnutrition. Pediatrics 2004;113:1549-58.

3. Chatfield S, Owen G, Ryley HC, et al. Neonatal screening for cystic fibrosis in Wales and the West Midlands: clinical assessment after five years of screening. Arch Dis Child 1991;66:29-33.

4. Siret D, Bretaudeau G, Branger B, et al. Comparing the clinical evolution of cystic fibrosis screened neonatally to that of cystic fibrosis diagnosed from clinical symptoms: a 10-year retrospective study in a French region (Brittany). Pediatr Pulmonol 2003;35:342-9.

5. Dankert-Roelse JE, te Meerman GJ, Martijn A, et al. Survival and clinical outcome in patients with cystic fibrosis, with or without neonatal screening. J Pediatr 1989;114:362-7.

6. Dankert-Roelse JE, Merelle ME. Review of outcomes of neonatal screening for cystic fibrosis versus non-screening in Europe. J Pediatr 2005;147:S15-20.

7. Merelle ME, Schouten JP, Gerritsen J, et al. Influence of neonatal screening and centralized treatment on long-term clinical outcome and survival of CF patients. Eur Respir J 2001;18:306-15.

8. Dijk FN, McKay K, Barzi F, et al. Improved survival in cystic fibrosis patients diagnosed by newborn screening compared to a historical cohort from the same centre. Arch Dis Child 2011;96:1118-23.

9. Linnane BM, Hall GL, Nolan G, et al. Lung function in infants with cystic fibrosis diagnosed by newborn screening. Am J Respir Crit Care Med 2008; 178:1238-44.

10. Pillarisetti $\mathbf{N}$, Williamson $\mathrm{E}$, Linnane $\mathrm{B}$, et al. Infection, inflammation and lung function decline in infants with cystic fibrosis. Am J Respir Crit Care Med 2011;184:75-81.
11. Ranganathan S, Dezateux CA, Bush $A$, et al. Airway function in infants newly diagnosed with cystic fibrosis. Lancet 2001;358:1964-5.

12. Ranganathan SC, Bush A, Dezateux C, et al. Relative ability of full and partial forced expiratory maneuvers to identify diminished airway function in infants with cystic fibrosis. Am J Respir Crit Care Med 2002;166:1350-7.

13. Aurora P, Stanojevic S, Wade A, et al. Lung clearance index at 4 years predicts subsequent lung function in children with cystic fibrosis. Am J Respir Crit Care Med 2011:183:752-8

14. Kozlowska WJ, Bush A, Wade A, et al. Lung function from infancy to the preschoo years after clinical diagnosis of cystic fibrosis. Am J Respir Crit Care Med 2008;178:42-9.

15. Ranganathan SC, Stocks J, Dezateux C, et al. The evolution of airway function in early childhood following clinical diagnosis of cystic fibrosis. Am J Respir Crit Care Med 2004;169:928-33.

16. Aurora $\mathbf{P}$, Bush A, Gustafsson $\mathrm{P}$, et al. Multiple-breath washout as a marker of lung disease in preschool children with cystic fibrosis. Am J Respir Crit Care Med 2005:171:249-56.

17. Gustafsson PM, Aurora P, Lindblad A. Evaluation of ventilation maldistribution as an early indicator of lung disease in children with cystic fibrosis. Eur Respir $J$ 2003;22:972-9.

18. Kraemer R, Blum A, Schibler A, et al. Ventilation inhomogeneities in relation to standard lung function in patients with cystic fibrosis. Am J Respir Crit Care Med 2005;171:371-8.

19. American Thoracic Society, European Respiratory Society. ATS/ERS statement: Raised volume forced expirations in infants: guidelines for current practice. Am J Respir Crit Care Med 2005;172:1463-71.

20. Lum S, Gustafsson P, Ljungberg $\mathrm{H}$, et al. Early detection of cystic fibrosis lung disease: multiple-breath washout versus raised volume tests. Thorax 2007;62:341-7.

21. Borowitz D, Robinson KA, Rosenfeld M, et al. Cystic Fibrosis Foundation evidence-based guidelines for management of infants with cystic fibrosis. J Pediatr 2009;155:S73-93.

22. Balfour-Lynn IM. Newborn screening for cystic fibrosis: evidence for benefit. Arch Dis Child 2008:93:7-10.

23. Report of the UK Cystic Fibrosis Trust Antibiotic Working Group. Antibiotic Treatment for Cystic Fibrosis. 3rd edn. Cystic Fibrosis Trust UK. 2009. http://www. cftrust.org.uk/aboutcf/publications/consensusdoc/Antibiotic_treatment_for_Cystic_ Fibrosis.pdf (accessed Feb 2012)

24. Equi AC, Pike SE, Davies J, et al. Use of cough swabs in a cystic fibrosis clinic. Arch Dis Child 2001;85:438-9.

25. Cole TJ, Wright CM, Williams AF. Designing the new UK-WHO growth charts to enhance assessment of growth around birth. Arch Dis Child Fetal Neonatal Ed 2012;97:F219-22.

26. Stocks J, Godfrey S, Beardsmore C, et al. Standards for infant respiratory function testing: plethysmographic measurements of lung volume and airway resistance. Eur Respir J 2001;17:302-12

27. Stocks J, Marchal F, Kraemer R, et al. Plethysmographic assessment of functional residual capacity and airway resistance. In: Stocks J, Sly PD, Tepper RS, et al, eds. Infant Respiratory Function Testing. New York: John Wiley \& Sons, 1996:191-240.

28. Goetz I, Hoo A-F, Lum S, et al. Assessment of passive respiratory mechanics in infants: double versus single occlusion? Eur Respir J 2001;17:449-55.

29. Aurora P, Kozlowska W. Stocks J. Gas mixing efficiency from birth to adulthood measured by multiple-breath washout. Respir Physiol Neurobiol 2005;148:125-39.

30. Ranganathan SC, Hoo AF, Lum SY, et al. Exploring the relationship between forced maximal flow at functional residual capacity and parameters of forced expiration from raised lung volume in healthy infants. Pediatr Pulmonol 2002;33:419-28.

31. Lum S, Sonnappa S, Gustafsson PM, et al. Lung growth and ventilation inhomogeneity in health. Eur Respir J 2011;38:351s.

32. Nguyen TTD, Hoo A-F, Lum $S$, et al. New reference equations to improve interpretation of infant lung function. Pediatr Pulmonol. In press.

33. Cohen J. Statistical Power Analysis for the Behavioural Sciences. 2nd edn. Hillsdale New Jersey: Erlbaum Associates, 1988.

34. Lum S, Hoo AF, Hulskamp G, et al. Potential misinterpretation of infant lung function unless prospective healthy controls are studied. Pediatr Pulmonol 2010;45:906-13

35. Stocks J, Modi N, Tepper R. Need for healthy controls when assessing lung function in infants with respiratory disease. Am J Respir Crit Care Med 2010;182:1340-2.

36. Gustafsson PM. Inert gas washout in preschool children. Paediatr Respir Rev 2005:6:239-45

37. Ranganathan $\mathbf{S}$, Goetz I, Hoo AF, et al. Assessment of tidal breathing parameters in infants with cystic fibrosis. Eur Respir J 2003;22:761-6.

38. Stocks J, Lum S. Pulmonary function tests in infants and preschool children. In: Chernick V, Bush A, eds. Kendig's disorders of the respiratory tract in children Philadelphia, PA, USA: Elsevier, 2012:169-210.

39. Armstrong DS, Grimwood K, Carlin JB, et al. Lower airway inflammation in infants and young children with cystic fibrosis. Am J Respir Crit Care Med 1997;156:1197-204.

40. Armstrong DS, Hook SM, Jamsen KM, et al. Lower airway inflammation in infants with cystic fibrosis detected by newborn screening. Pediatr Pulmonol 2005:40:500-10.

41. Khan TZ, Wagener JS, Bost T, et al. Early pulmonary inflammation in infants with cystic fibrosis. Am J Respir Crit Care Med 1995;151:1075-82. 
42. Davis SD, Rosenfeld M, Kerby GS, et al. Multicenter evaluation of infant lung function tests as cystic fibrosis clinical trial endpoints. Am J Respir Crit Care Med 2010;182:1387-97.

43. Gustafsson PM, Kallman S, Ljungberg H, et al. Method for assessment of volume of trapped gas in infants during multiple-breath inert gas washout. Pediatr Pulmonol 2003; 35:42-9.

44. Gustafsson PM, de Jong PA, Tiddens HA, et al. Multiple-breath inert gas washout and spirometry versus structural lung disease in cystic fibrosis. Thorax 2008;63:129-34.
45. Jones M, Castile R, Davis $\mathbf{S}$, et al. Forced expiratory flows and volumes in infants: normative data and lung growth. Am J Respir Crit Care Med 2000;161:353-9.

46. Kerem E, Hirawat S, Armoni S, et al. Effectiveness of PTC124 treatment of cystic fibrosis caused by nonsense mutations: a prospective phase II trial. Lancet 2008; 372:719-27.

47. Ramsey BW, Davies J, McElvaney NG, et al. A CFTR potentiator in patients with cystic fibrosis and the G551D mutation. N Engl J Med 2011;365:1663-72 